\section{Proton therapy for pediatric cancer: are we ready for prime time?}
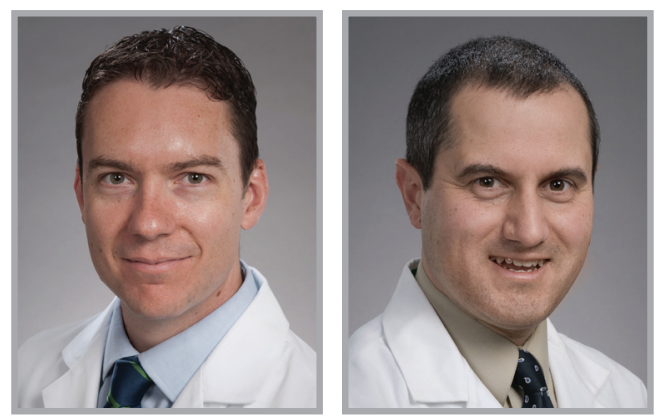

“...it could be very challenging to accrue a pediatric randomized clinical trial between photon therapy and proton beam therapy when families are informed that a randomization could result in their children receiving higher integral radiation doses to healthy tissues."

Tobias R Chapman' \& Ralph P Ermoian*,1

First draft submitted: 7 August 2016; Accepted for publication: 23 August 2016; Published online: 22 September 2016

The majority of pediatric oncology patients become long-term survivors who must live with the effects of their diseases and treatments for many decades [1]. Although there is a trend toward reduced use of radiation therapy in children with cancer, it continues to play an essential role in the treatment of many common pediatric malignancies, including brain tumors, sarcomas, lymphomas and neuroblastoma [2]. Therefore, it is imperative to deliver effective radiation therapy with the least morbidity possible in the treatment of children with cancer. Proton beam therapy (PBT) is promising for its potential to provide equivalent efficacy in treating pediatric cancer patients with improved acute and late side-effect profiles due to the lack of exit dose from its beams.

Most pediatric cancer patients in the USA (and worldwide) require radiotherapy receive photon (or electron) radiation, however, the use of PBT is growing. Fifteen years ago, there were two clinical PBT facilities, both treating pediatric patients in the USA; now there are 23 PBT centers, the majority of which treat children [3]. More pediatric cancer patients are being treated with PBT, including an increase of 33\% between 2010 and 2012 [4].

\section{Data supporting PBT in pediatric cancers}

With its increased use, the data supporting PBT for pediatric cancers merit examination. In most cases, patients are prescribed the same doses of radiation as they would receive with photons; there have not been multi-institution or cooperative group PBT dose-escalation trials for pediatric tumors. In fact, on Children's Oncology Group trials that allow PBT, the radiation modality is determined by the treating physician and identical prescription doses and organs at risk dose constraints are used.

To date, no randomized clinical trials of PBT versus photon radiotherapy have been conducted for pediatric cancers. The data supporting PBT are largely drawn from dosimetric studies, retrospective reviews of single institution cohorts and (mostly) single institution Phase II studies. Dosimetric studies of PBT craniospinal irradiation

'Radiation Oncology, University of Washington, Seattle, WA, USA

*Author for correspondence: Tel.: +1 206598 4100; Fax: +1 206598 3786; ralphpe@uw.edu

\section{KEYWORDS}

- efficacy $\bullet$ pediatric cancer $\bullet$ proton radiotherapy

\section{“The majority of pediatric oncology patients become long-term survivors who must live with the effects of their diseases and treatments for many decades."}

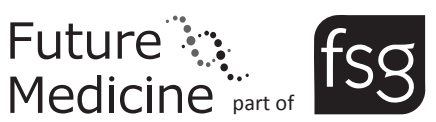


“Cooperative groups need to either develop proton beam therapy-specific Phase II trials, or (at least) prospectively collect the treatment and outcome data to perform planned subset analyses between modalities of radiation.” have demonstrated reduced dose to organs at risk distal to the target that would be expected to result in decreased late risks of infertility and ovarian failure [5], heart disease [6] and secondary breast cancer [7], among other benefits. Similar dosimetric studies for lymphoma treatment show a potential for reduced risk of heart disease [8], and studies in CNS tumors suggest reduced neurocognitive effects with PBT [9].

A number of case series and Phase II studies demonstrate at least comparable efficacy of PBT to recent clinical trials with photon radiation, but with favorable side-effect profiles. Studies with limited numbers of PBT patients were initially published in the 1990s, but the rate of publications has accelerated as proton centers have developed more experience. A critical review by Leroy et al. summarized the pediatric PBT studies with the highest quality data [10]. Highlights from recent studies include equivalent efficacy to photon radiotherapy in medulloblastoma [11], very low rates of audiologic and endocrine effects from PBT of ependymoma [12], good quality of life following PBT treatment of CNS tumors [13], and improved gastrointestinal and hematologic toxicity when treating with PBT craniospinal irradiation [14]. Promising as these results are, the obvious critique is that they are almost exclusively single-institution studies, and many are retrospective case series, subject to concerns regarding selection bias. To date, cooperative groups such as the Children's Oncology Group have not published unplanned subset analyses of recent studies comparing patients treated with the two modalities.

\section{Cost-effectiveness}

Concerns have been raised about cost-effectiveness. PBT is more cost-intensive than photon therapy, particularly when treating children [15]. Two of the first PBT cost-effectiveness studies were for CNS tumors in children. In studies from investigators at Massachusetts General Hospital, PBT is cost effective in treating medulloblastoma due to the decreased anticipated cost of late effects [16], and in treating CNS tumors when PBT results in incremental protection of pituitary gland production of growth hormone [17]. Other investigators have modeled cost savings associated with cochlea sparing with PBT [18].

\section{Clinical concerns}

While there are promising toxicity profiles with PBT, there are two critical toxicity questions: Is there an increased incidence of secondary malignancies related to neutron contamination, and are there increased normal tissue effects from relative biologic effectiveness (RBE) uncertainty?

There is concern that the number of secondary malignancies from generation of neutron contamination during treatment could more than offset the decreases from reduced radiation exposure distal to target volumes [19]. This is thought to be of particular concern in passive scattering and uniform scanning systems including when the beam is produced by scattering foils and brass apertures, processes that generate much of the neutron contamination [20]. In spite of theoretical concerns, the clinical data have yet to show an increased risk of secondary malignancy with PBT. A matched cohort analysis including PBT and photon patients did not find a significant difference in secondary malignancy rates, with a trend toward fewer secondary malignancy rates in patients treated with PBT [21]. In addition, a study of patients treated for retinoblastoma found that PBT was associated with lower rates of secondary malignancy [22], and of 313 patients reported in six series of pediatric patients-treated PBT at Massachusetts General Hospital, only six developed solid tumor secondary malignancies, with four hematologic malignancies [23]. Pencil-beam scanning PBT, recently deployed at several proton centers, generates less neutron contamination [20].

Also of clinical concern is RBE uncertainty and associated possible increased incidence of CNS toxicity. Researchers from MD Anderson and Baylor found that the rate of imaging changes in ependymoma patients after radiation therapy is higher in PBT patients than photon patients, although the rate of symptomatic imaging changes was similar [24]. Massachusetts General Hospital has recently reported its experience in medulloblastoma patients and found rates of symptomatic radiation injury of only $2.7 \%$ [25]. However, Indelicato et al. recently reported a greater than $10 \%$ risk of grade 2 or greater brainstem injury in pediatric PBT patients treated for posterior fossa tumors, with a maximum brainstem point dose greater than 56.5 cobalt Gray equivalent (CGE), brainstem D50\% more than 52.3 CGE or brainstem D10\% more than 55.3 CGE [26]. These dose levels are commonly exceeded when treating with photon therapy, and pediatric radiation oncologists are increasingly using the dose constraints suggested by this study in their radiation 
planning. Ongoing research has focused on dose modeling, RBE uncertainty and increased linear energy transfer as possible explanations for these toxicities [25].

\section{Looking ahead}

Additional proton centers are planned throughout the world and some countries such as the UK and Canada send selected patients out of country to receive PBT. The number of pediatric patients receiving $\mathrm{PBT}$ is growing.

The available PBT literature reveals a modality that provides effective oncologic outcomes with favorable side-effect profiles, and is appropriate to be used in many but not all cases. No study has shown decreased efficacy with PBT, and there does not appear to be an increased risk of secondary malignancies. The data from Indelicato et al. [26] have reinforced the need to be conservative in dose constraints until further data are available. Radiation oncologists may choose not to offer PBT to patients whose treatment cannot meet the more conservative dose constraints, and that is reasonable. PBT-specific dose constraints are being considered either for modifications of existing pediatric trials or for future trials.

In an ideal world, one would perform large randomized clinical trials to provide adequate power to fully investigate PBT versus photon therapy. This has not been the standard applied before adopting other new radiation techniques including 3D conformal therapy, intensitymodulated radiation therapy, stereotactic body radiosurgery and volumetric arc radiotherapy. Practically speaking, it could be very challenging to accrue a pediatric randomized clinical trial between photon therapy and PBT when families are informed that a randomization could result in their children receiving higher integral radiation doses to healthy tissues.

Reassuring as so much of the data is, we must continue to analyze the clinical data already obtained and collect more to further quantify efficacy and risks. Cooperative group trials that have allowed PBT need to perform unplanned subset analyses of PBT versus photon therapy outcomes. At a minimum, patients' families should be offered participation on the multi-institution registry trials; the Pediatric Proton Consortium and Proton Cooperative Group registry trials have enrolled hundreds of patients. Institutions need to partner to offer Phase II studies by disease sites. Cooperative groups need to either develop PBT-specific Phase II trials, or (at least) prospectively collect the treatment and outcome data to perform planned subset analyses between modalities of radiation.

\section{Financial \& competing interests disclosure}

RP Ermoian is employed at the University of Washington where proton therapy is a treatment option; however, he receives no financial incentives for choosing one radiation modality (e.g., proton radiation therapy) over another. The authors have no other relevant affliations or financial involvement with any organization or entity with a financial interest in or financial conflict with the subject matter or materials discussed in the manuscript apart from those disclosed.

No writing assistance was utilized in the production of this manuscript.

\section{References}

Papers of special note have been highlighted as:

- of interest; $\bullet$ of considerable interest

1 Siegel R, DeSantis C, Virgo Ket al. Cancer treatment and survivorship statistics, 2012. CA Cancer J. Clin. 62(4), 220-241 (2012).

2 Jairam V, Roberts KB, Yu JB. Historical trends in the use of radiation therapy for pediatric cancers: 1973-2008. Int. J. Radiat. Oncol. Biol. Phys. 85(3), e151-e155 (2013).

- Shows the decreasing use of radiation therapy in treating pediatric cancer patients.

3 The National Association for Proton Therapy. www.proton-therapy.org/map.htm

4 Chang AL, Yock TI, Mahajan A et al. Pediatric proton therapy: patterns of care across the United States. Int. J. Particle Ther. 1(2), 357-367 (2014).

- This is the best published data on the increasing use of proton beam therapy (PBT) in treating pediatric cancer patients.

5 Lester-Coll NH, Morse CB, Zhai HA et al. Preserving fertility in adolescent girls and young women requiring craniospinal irradiation: a case report and discussion of options to be considered prior to treatment. J. Adolesc. Young Adult Oncol. 3(2), 96-99 (2014).

6 Zhang R, Howell RM, Taddei PJ et al. A comparative study on the risks of radiogenic second cancers and cardiac mortality in a set of pediatric medulloblastoma patients treated with photon or proton craniospinal irradiation. Radiother. Oncol. 113, 84-88 (2014).

7 Kumar RJ, Zhai H, Both S, Tochner Z, Lustig R, Hill-Kayser C. Breast cancer screening for childhood cancer survivors after craniospinal irradiation with protons versus x-rays: a dosimetric analysis and review of the literature. Pediatr. Hematol. Oncol. 35, 462-467 (2013).

8 Hoppe BS, Flampouri S, Su Z et al. Effective dose reduction to cardiac structures using protons compared with 3DCRT and IMRT in mediastinal Hodgkin lymphoma. Int. J. Radiation Oncol. Biol. Phys. 84(2), e449-e455 (2012).

9 Merchant TE, Hua CH, Shukla H, Ying X, Nill S, Oelfke U. Proton versus photon 
radiotherapy for common pediatric brain tumors: comparison of models of dose characteristics and their relationship to cognitive function. Pediatr. Blood Cancer 51(1), 110-117 (2008).

10 Leroy R, Benahmed N, Hulstaert F, Van Damme N, De Ruysscher D. Proton therapy in children: a systematic review of clinical effectiveness in 15 pediatric cancers. Int. J. Radiat. Oncol. Biol. Phys. 95(1), 267-278 (2016).

- Presents a systematic assessment of the quality of data available about treating children with PBT.

11 Eaton BR, Esiashvili N, Kim S et al. Clinical outcomes among children with standard-risk medulloblastoma treated with proton and photon radiation therapy: a comparison of disease control and overall survival. Int. J. Radiat. Oncol. Biol. Phys. 94(1), 133-138 (2016).

- Shows equivalency in efficacy between proton and photon therapy in treating medulloblastoma.

12 Macdonald SM, Sethi R, Lavally B et al. Proton radiotherapy for pediatric central nervous system ependymoma: clinical outcomes for 70 patients. Neuro Oncol. 15(11), 1552-1559 (2013).

13 Yock TI, Bhat S, Szymonifka J et al. Quality of life outcomes in proton and photon treated pediatric brain tumor survivors. Radiother. Oncol. 113(1), 89-94 (2014).

14 Song S, Park HJ, Yoon JH et al. Proton beam therapy reduces the incidence of acute haematological and gastrointestinal toxicities associated with craniospinal irradiation in pediatric brain tumors. Acta Oncol. 53(9), 1158-1164 (2014).

15 Johnstone PA, Kerstiens J. Doing poorly by doing good: the bottom line of proton therapy for children. J. Am. Coll. Radiol. 11(10), 995-997 (2014).

16 Mailhot Vega RB, Kim J, Bussière M et al. Cost effectiveness of proton therapy compared with photon therapy in the management of pediatric medulloblastoma. Cancer 119(24), 4299-4307 (2013).

17 Mailhot Vega R, Kim J, Hollander A et al. Cost effectiveness of proton versus photon radiation therapy with respect to the risk of growth hormone deficiency in children. Cancer 121(10), 1694-1702 (2015).

18 Hirano E, Fuji H, Onoe T, Kumar V, Shirato $\mathrm{H}$, Kawabuchi K. Cost-effectiveness analysis of cochlear dose reduction by proton beam therapy for medulloblastoma in childhood. J. Radiat. Res. 55(2), 320-327 (2014).

19 Brenner DJ, Hall EJ. Secondary neutrons in clinical proton radiotherapy: a charged issue. Radiother. Oncol. 86, 165-170 (2008).

20 Proton Therapy Physics. Paganetti H (Ed.). Taylor \& Francis/CRC Press, Boca Raton, FL, USA (2012).

21 Chung CS, Yock TI, Nelson K, Xu Y, Keating NL, Tarbell NJ. Incidence of second malignancies among patients treated with proton versus photon radiation. Int. J. Radiat. Oncol. Biol. Phys. 87(1), 46-52 (2013).
22 Sethi RV, Shih HA, Yeap BY, Mouw KW et al. Second nonocular tumors among survivors of retinoblastoma treated with contemporary photon and proton radiotherapy. Cancer 120(1), 126-133 (2014).

23 Eaton BR, MacDonald SM, Yock TI, Tarbel NJ. Secondary malignancy risk following proton radiation therapy. Front. Oncol. 5, 261 (2015).

-• Provides the most comprehensive clinical data about secondary malignancies associated with $\mathrm{PBT}$ at one institution.

24 Gunther JR, Sato M, Chintagumpala M et al. Imaging changes in pediatric intracranial ependymoma patients treated with proton beam radiation therapy compared with intensity modulated radiation therapy. Int. J. Radiat. Oncol. Biol. Phys. 93(1), 54-63 (2015).

25 Giantsoudi D, Sethi RV, Yeap BY et al. Incidence of CNS injury for a cohort of 111 patients treated with proton therapy for medulloblastoma: LET and RBE associations for areas of injury. Int. J. Radiat. Oncol. Biol. Phys. 95(1), 287-296 (2016).

- Provides reassuring data regarding CNS injury in treating medulloblastoma with PBT.

26 Indelicato DJ, Flampouri S, Rotondo RL et al. Incidence and dosimetric parameters of pediatric brainstem toxicity following proton therapy. Acta Oncol. 53(10), 1298-1304 (2014).

- Provides dosimetric criteria associated with PBT-associated brainstem injuries that many pediatric radiation oncologists now use in planning PBT in children. 